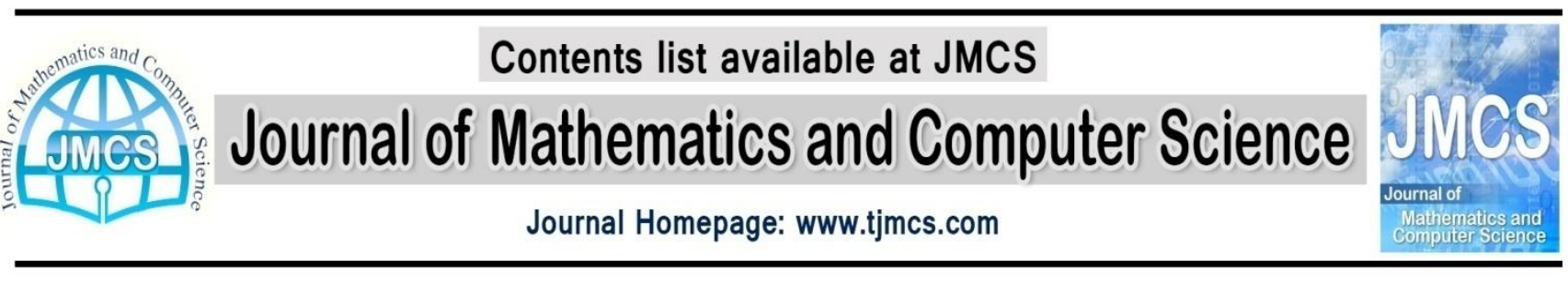

\title{
Single Model Approach to Evaluate Optimistic and Pessimistic Congestion in Fuzzy Data Envelopment Analysis
}

\author{
Fatemeh Baghbani Abootaleb ${ }^{1}$, Alireza Alinezhad ${ }^{1}$, Mehdi Yazdani ${ }^{1, *}$ \\ ${ }^{1}$ Faculty of Industrial and Mechanical Engineering, Qazvin Branch, Islamic Azad University, Qazvin, Iran \\ sally_baghbani@yahoo.com,alinezhad_ir@yahoo.com, mehdi_yazdani2007@yahoo.com
}

Article history:

Received December 2013

Accepted January 2014

Available online February 2014

\begin{abstract}
Data Envelopment Analysis (DEA) is an almost new data oriented approach for measuring the performance of series of homogenous entities named Decision Making Unit (DMU) with the ability to alter multiple inputs into multiple outputs. This article investigates the development of congestion model with regard to fuzzy input and output. Also, a new concept called as optimistic and pessimistic are introduced for congestion model. To create this concept, the upper and lower bound of efficiency, outputs and inputs are required. This concept is provided with $\alpha$-cut approach in fuzzy data envelopment analysis (FDEA). In order to evaluate the efficiency of given models, data of some universities of Iran are applied. The results reveal the proper validity of proposed model.
\end{abstract}

Keywords: Data envelopment analysis, fuzzy inputs and outputs, $\alpha$-cut approach, optimistic and pessimistic congestion.

\section{Introduction}

Date Envelopment Analysis (DEA) means a methodological programming approach for evaluating relative efficiencies within a group of Decision Making Units (DMUs). The definition of DMU is generic and flexible. We have seen a great many applications of DEA in recent years to be used in measuring the performances of various entities involved in numerous activities in a lot of different contexts in many different countries. We have used DMUs of various forms to measure the performance of entities, for example, hospitals, universities, business firms and so on, and also the performance of countries or regions. DEA which is a very helpful management and decision making tool has experienced surprising developments in both methodology and theory. Early traditional DEA models (for instance, CCR (Charnes, Cooper, Rhodes) [1] and BCC (Banker, Cooper, Charnes)[2] did not consider imprecise data and supposed that all input and output data are exactly known. However, in a real situation, this supposition does not always seem to be true.

\footnotetext{
*Corresponding Author
} 
In a general sense, uncertain information of imprecise data can be shown in interval or fuzzy numbers. DEA is one of the efficient and useful methods to determine the efficiency of DMUs. The main feature of this method is that the DMUs apply the same inputs to gain the same outputs and this is the very feature which makes the units comparable [3]. In the classical models it is assumed that the information of all inputs and outputs is completely precise and crisp. However, this assumption may not be true in the real world. In the research which has been done so far, the classical efficiency evaluation methods are used which include the CCR and BCC methods [4]. Since there is some risk involved, these methods do not seem to be proper for many sections. Cooper et.al [5] and Zhu [6] applied the concept of risk to the inputs and outputs using the fuzzy concept in data envelopment analysis. The imprecise models of DEA are various and this is defined as stochastic models, interval models, and fuzzy models. In this article, the last one is used to clarify the imprecise inputs and outputs. Congestion is introduced as an economic concept which is known as inefficiency. However, this inefficiency is totally different from technical inefficiency. "Evidence of congestion is presented when reduction in one or more inputs can be associated with increase in one or more outputs- without worsening any other input or output. Proceeding in reverse, congestion occurs when increase in one or more inputs can be associated with decrease is one or more outputs- without improving any other input or output." [5].The inefficiency, of course, occurs when it is possible to improve some inputs and outputs without worsening the other inputs or outputs. But congestion in inputs comes along with an improvement in at least one of the outputs [7]. Recognizing and calculation of DMUs with congestion has two advantages. First, if this DMU is eliminated, since it is related to input and input is related to cost, the cost will decrease. Second, congestion decreases outputs, so with eliminating congestion, the output will increase.

The remainder of this paper is organized as follows: in section 2 we developed a new congestion method on the basis of Cooper et al. Section 3 introduce both pessimistic and optimistic congestion.Section 4 measures congestion in a real case which is related to 23 Iranian universities. By using the proposed methodology, we solve several numerical examples from the literature on congestion. In section 5, we study the data in tables and draw a conclusion.

\section{Literature Review}

Among all methods proposed to evaluate congestion in DEA, there are two main methods called FGL (Fare, Grroskopf, Lovell) [8] and CTT (Cooper,Thompson,Thrall) [9]. The FGL model is the first proposed method to calculate congestion which can only recognize the presence or absence of congestion.

In CTT method, the value of congestion can be measured. In this method sometimes technical efficiency is mistaken for congestion [10]. To illustrate the applications of congestion, the practical research by Broket et al. [11] were conducted to recognize the congestion problem in China. In CTT model, the congestion is determined by the proportion of the observed value to the expected value. Later, researchers in several studies compared the FGL and CTT models and they concluded that the CTT method had some advantages to FGL. Broket et al. [11] modified the CTT method which later became known as BCSW method. Cooper et al. [12] developed CTT method to a method with an integrated additive model. Also, Cooper et al. [5] Proposed a method with a single model. The main idea of current article is based upon this model. Another method with two models were introduced by Tone and Saho [13]. In this model, firstly the pure technical efficiency is calculated and then the technical efficiency. Then value of congestion is determined based on the proportion of pure technical efficiency to technical efficiency. Jahanshahloo and Khodabakhshi [14] proposed a method with two models for congestion. Khodabakhshi [15] suggested a method with single model in the form of a suitable combination of input relaxation to evaluate congestion of input. Fanati [16] recommended a non- radial method to measure congestion. 
In all the researches done so far about congestion, the data used were crisp whereas, and in the majority of cases, the given data are imprecise. Hence, a need for implementing imprecise data and utilizing the fuzzy methods for the evaluation of congestion value seem inevitable.

The concept of fuzzy was first introduced by LotfiAsgarzadeh [17] published in his article titled as "Fuzzy Sets" which discussed the probable problems in making models for systems. The fuzzy theory with imprecise data is applicable in DEA.

Fuzzy DEA is used to measure performance evaluation in organization with uncertain and blurred variables. There exist different researches on fuzzy DEA in the related literature.

Cooper et al. [18] proposed a model with the power to consider the inputs and outputs as crisp and interval numbers. Sengupta was the first to apply fuzzy programming problem method to DEA models in 1992 for the units with several inputs and one output.Another method for FDEA was introduced by Geo and Tanaka in 2001 in which the data are considered as fuzzy, triangular, and symmetrical numbers and after applying the $\alpha$-cut approach to the constraints, they managed to determine efficiency for each DMU by solving two linear programing problems [18]. Saati et al. proposed a method by which DEA models can be solved by fuzzy numbers [19]. In their method, it is possible to use crisp numbers and every fuzzy number as input and output.

By studying the related literature we can conclude that no research has been done about the evaluation of congestion in the fuzzy state. Therefore we are going to deal with this issue in this article.

\section{Proposed method:}

Suppose there are 'n' decision making units with 'm' inputs and 's' outputs where $x_{i j}=\left(x_{1}, x_{2} \ldots . x_{m}\right)$ and $y_{r j}=\left(y_{1}, y_{2}, \ldots y_{s}\right)$ are the inputs and outputs of decision making units and in fuzzy case the inputs and outputs are fuzzy numbers. With these inputs and outputs in the form of $\tilde{x}_{i j}=\left(x_{i j}^{l}, x_{i j}^{m}, x_{i j}^{u}\right)$ and $\tilde{y}_{r j}=\left(y_{r j}^{l}, y_{r j}^{m}, y_{r j}^{u}\right)$ congestion is measured.

$$
\begin{gathered}
\min \sum_{\mathrm{i}=1}^{\mathrm{m}} \mathrm{s}_{\mathrm{i}}^{-\mathrm{c}} \quad(1) \\
\mathrm{s} . \mathrm{t}: \\
\sum_{\mathrm{j}=1}^{\mathrm{n}} \lambda_{\mathrm{j}} \tilde{\mathrm{x}}_{\mathrm{ij}}+\mathrm{s}_{\mathrm{i}}^{-\mathrm{c}}=\tilde{\mathrm{x}}_{\mathrm{i} \mathrm{i}}, \mathrm{i}=1,2, \ldots, \mathrm{m} \\
\sum_{\mathrm{j}=1}^{\mathrm{n}} \lambda_{\mathrm{j}} \tilde{\mathrm{y}}_{\mathrm{rj}}=\tilde{\mathrm{y}}_{\mathrm{ro}} \varphi^{*}+\mathrm{s}_{\mathrm{r}}{ }^{+}, \mathrm{r}=1,2, \ldots, \mathrm{s} \\
\sum_{j=1}^{n} \lambda_{j}=1 \\
\lambda_{j}, s_{i}^{-c} \geq 0 \\
i=1,2, \ldots, m, r=1,2, \ldots, s, j=1,2, \ldots, n
\end{gathered}
$$

Where $s_{i}^{-c}$ the amount of congestion is, $\varphi^{*}$ is the amount of efficiency. 


$$
\begin{gathered}
\max \varphi(2) \\
\text { s.t : } \\
\sum_{\mathrm{j}=1}^{\mathrm{n}} \lambda_{\mathrm{j}} \tilde{x}_{\mathrm{ij}} \leq \tilde{\mathrm{x}}_{\mathrm{io}} \quad, \mathrm{i}=1,2, \ldots \mathrm{m} \\
\sum_{\mathrm{j}=1}^{\mathrm{n}} \lambda_{\mathrm{j}} \tilde{\mathrm{y}}_{\mathrm{rj}} \geq \tilde{\mathrm{y}}_{\mathrm{ro}} \varphi \quad, \mathrm{r}=1,2, \ldots \mathrm{s} \\
\sum_{\mathrm{j}=1}^{\mathrm{n}} \lambda_{\mathrm{j}}=1 \\
\lambda_{\mathrm{j}} \geq 0,, \mathrm{i}=1,2, \ldots \mathrm{m}, \mathrm{r}=1,2, \ldots \mathrm{s}, \mathrm{j}=1,2, \ldots \mathrm{n}
\end{gathered}
$$

With respect to $s_{r}^{+}, s_{i}^{-c}$ slacks, model (2) will turn to an equivalence relation as shown in model (3):

$$
\begin{gathered}
\max \varphi+\left(\sum_{\mathrm{r}=1}^{\mathrm{s}} \mathrm{s}_{\mathrm{r}}^{+}-\sum_{\mathrm{i}=1}^{\mathrm{m}} \mathrm{s}_{\mathrm{i}}^{-\mathrm{c}}\right) \quad(3) \\
\mathrm{s.t}: \quad(15) \\
\sum_{\mathrm{j}=1}^{\mathrm{n}} \lambda_{\mathrm{j}} \tilde{\mathrm{x}}_{\mathrm{ij}}+\mathrm{s}_{\mathrm{i}}^{-\mathrm{c}}=\tilde{\mathrm{x}}_{\mathrm{i} \mathrm{o}} \quad, \mathrm{i}=1,2, \ldots, \mathrm{m} \\
\sum_{\mathrm{j}=1}^{\mathrm{n}} \lambda_{\mathrm{j}} \tilde{\mathrm{y}}_{\mathrm{rj}}-\mathrm{s}_{\mathrm{r}}^{+}=\tilde{y}_{\mathrm{ro}} \varphi, \mathrm{r}=1,2, \ldots, \mathrm{s} \\
\sum_{\mathrm{j}=1}^{\mathrm{n}} \lambda_{\mathrm{j}}=1 \\
\lambda_{\mathrm{j}} \geq 0, \mathrm{~s}_{\mathrm{r}}^{+} \geq 0, \mathrm{~s}_{\mathrm{i}}^{-} \geq 0, \\
\mathrm{i}=1,2, \ldots, \mathrm{m}, \mathrm{r}=1,2, \ldots, \mathrm{s}, \mathrm{j}=1,2, \ldots, \mathrm{n}
\end{gathered}
$$

Model(3) can be solved in two stages. The first of which is obtained from BCC output oriented model and the amount of efficiency will then be obtained from model(4):

$$
\begin{gathered}
\max \varphi \quad(4) \\
\text { s.t : } \\
\sum_{\mathrm{j}=1}^{\mathrm{n}} \lambda_{\mathrm{j}}\left(\mathrm{x}_{\mathrm{ij}}^{1}, \mathrm{x}_{\mathrm{ij}}^{\mathrm{m}}, \mathrm{x}_{\mathrm{ij}}^{\mathrm{u}}\right) \leq\left(\mathrm{x}_{\mathrm{io}}^{1}, \mathrm{x}_{\mathrm{io}}^{\mathrm{m}}, \mathrm{x}_{\mathrm{io}}^{\mathrm{u}}\right), \mathrm{i}=1,2, \ldots, \mathrm{m} \\
\sum_{\mathrm{j}=1}^{\mathrm{n}} \lambda_{\mathrm{j}}\left(\mathrm{y}_{\mathrm{rj}}^{1}, \mathrm{y}_{\mathrm{rj}}^{\mathrm{m}}, \mathrm{y}_{\mathrm{rj}}^{\mathrm{u}}\right) \geq \varphi\left(\mathrm{y}_{\mathrm{ro}}^{1}, \mathrm{y}_{\mathrm{ro}}^{\mathrm{m}}, \mathrm{y}_{\mathrm{ro}}^{\mathrm{u}}\right), \mathrm{r}=1,2, \ldots, \mathrm{s} \\
\sum_{\mathrm{j}=1}^{\mathrm{n}} \lambda_{\mathrm{j}}=1 \\
\lambda_{\mathrm{j}} \geq 0, \quad \mathrm{i}=1,2, \ldots, \mathrm{m}, \mathrm{r}=1,2, \ldots, \mathrm{s}, \mathrm{j}=1,2, \ldots, \mathrm{n}
\end{gathered}
$$

Using $\alpha$ - cut approach, model (4) will be changed to model (5) and then model (6): 


$$
\begin{gathered}
\max \varphi \\
\text { s.t: } \\
\sum_{j=1}^{\mathrm{n}} \lambda_{\mathrm{j}}\left(\alpha \mathrm{x}_{\mathrm{ij}}^{\mathrm{m}}+(1-\alpha) \mathrm{x}_{\mathrm{ij}}^{1}, \alpha \mathrm{x}_{\mathrm{ij}}^{\mathrm{m}}+(1-\alpha) \mathrm{x}_{\mathrm{ij}}^{\mathrm{u}}\right) \leq \\
\left(\alpha \mathrm{x}_{\mathrm{io}}^{\mathrm{m}}+(1-\alpha) \mathrm{x}_{\mathrm{io}}^{1}, \alpha \mathrm{x}_{\mathrm{io}}^{\mathrm{m}}+(1-\alpha) \mathrm{x}_{\mathrm{io}}^{\mathrm{u}}\right), \mathrm{i}=1,2, \ldots, \mathrm{m} \\
\sum_{\mathrm{j}=1}^{\mathrm{n}} \lambda_{\mathrm{j}}\left(\alpha \mathrm{y}_{\mathrm{rj}}^{\mathrm{m}}+(1-\alpha) \mathrm{y}_{\mathrm{rj}}^{1}, \alpha \mathrm{y}_{\mathrm{rj}}^{\mathrm{m}}+(1-\alpha) \mathrm{y}_{\mathrm{rj}}^{\mathrm{u}}\right) \geq \\
\varphi\left(\alpha \mathrm{y}_{\mathrm{ro}}^{\mathrm{m}}+(1-\alpha) \mathrm{y}_{\mathrm{ro}}^{1}, \alpha \mathrm{y}_{\mathrm{ro}}^{\mathrm{m}}+(1-\alpha) \mathrm{y}_{\mathrm{ro}}^{\mathrm{u}}\right), \mathrm{r}=1,2, \ldots, \mathrm{s} \\
\sum_{\mathrm{j}=1}^{\mathrm{n}} \lambda_{\mathrm{j}}=1 \\
\lambda_{\mathrm{j}} \geq 0, \quad \mathrm{i}=1,2, \ldots, \mathrm{m}, \mathrm{r}=1,2, \ldots, \mathrm{s}, \mathrm{j}=1,2, \ldots, \mathrm{n}
\end{gathered}
$$

Having upper and lower bound for the first stage, there will be the following form:

$$
\begin{gathered}
\max \varphi(6) \\
s . t: \\
\sum_{j=1}^{n} \lambda_{j}\left[\underline{x}_{i j}, \bar{x}_{i j}\right] \leq\left[\underline{x}_{i o}, \bar{x}_{i o}\right], i=1,2, \ldots, m \\
\sum_{j=1}^{n} \lambda_{j}\left[\underline{y}_{r j}, \bar{y}_{r j}\right] \geq \varphi\left[\underline{y}_{r o}, \bar{y}_{r o}\right], r=1,2, \ldots, s \\
\sum_{j=1}^{n} \lambda_{j}=1 \\
\lambda_{j} \geq 0, i=1,2, \ldots, m, r=1,2, \ldots, s, j=1,2, \ldots, n
\end{gathered}
$$

$\bar{\varphi}_{o}$ is the amount which is obtained from model (7) and then is inserted within the model (8).

$$
\begin{gathered}
\max \bar{\varphi}_{\mathrm{o}}(7) \\
\text { s.t : } \\
\sum_{\mathrm{j}=1}^{\mathrm{n}} \lambda_{\mathrm{j}} \overline{\mathrm{x}}_{\mathrm{ij}} \leq \underline{\mathrm{x}}_{\mathrm{io}}, \mathrm{i}=1,2, \ldots, \mathrm{m} \\
\sum_{\mathrm{j}=1}^{\mathrm{n}} \lambda_{\mathrm{j}} \underline{\mathrm{y}}_{\mathrm{rj}} \geq \varphi \overline{\mathrm{y}}_{\mathrm{ro}}, \mathrm{r}=1,2, \ldots, \mathrm{s} \\
\sum_{\mathrm{j}=1}^{\mathrm{n}} \lambda_{\mathrm{j}}=1 \\
\lambda_{\mathrm{j}} \geq 0, \quad \mathrm{i}=1,2, \ldots, \mathrm{m}, \mathrm{r}=1,2, \ldots, \mathrm{s}, \mathrm{j}=1,2, \ldots, \mathrm{n}
\end{gathered}
$$

Finally model (8) represent the amount of $s_{i}^{-o c}$ which is the optimistic congestion. 


$$
\begin{gathered}
\max \left(\sum_{\mathrm{r}=1}^{\mathrm{s}} \mathrm{s}_{\mathrm{r}}^{+}-\sum_{\mathrm{i}=1}^{\mathrm{m}} \mathrm{s}_{\mathrm{i}}^{-\mathrm{oc}}\right) \\
\mathrm{s.t}: \quad(45) \\
\sum_{\mathrm{j}=1}^{\mathrm{n}} \lambda_{\mathrm{j}} \overline{\mathrm{x}}_{\mathrm{ij}}+\mathrm{s}_{\mathrm{i}}^{-\mathrm{oc}}=\underline{\mathrm{x}}_{\mathrm{io}} \quad, \mathrm{i}=1,2, \ldots, \mathrm{m} \\
\sum_{\mathrm{j}=1}^{\mathrm{n}} \lambda_{\mathrm{j}} \underline{\mathrm{y}}_{\mathrm{rj}}-\mathrm{s}_{\mathrm{r}}^{+}=\overline{\mathrm{y}}_{\mathrm{ro}} \bar{\varphi}_{\mathrm{o}}^{*}, \mathrm{r}=1,2, \ldots, \mathrm{s} \\
\sum_{\mathrm{j}=1}^{\mathrm{n}} \lambda_{\mathrm{j}}=1 \\
\lambda_{\mathrm{j}} \geq 0, \mathrm{~s}_{\mathrm{r}}^{+} \geq 0, \mathrm{~s}_{\mathrm{i}}^{-\mathrm{oc}} \geq 0 \\
\mathrm{i}=1,2, \ldots, \mathrm{m}, \mathrm{r}=1,2, \ldots, \mathrm{s}, \mathrm{j}=1,2, \ldots, \mathrm{n}
\end{gathered}
$$

The lower bound of efficiency is given below:

$$
\begin{gathered}
\max \underline{\varphi}_{o} \quad(9) \\
\text { s.t }: \\
\sum_{j=1}^{n} \lambda_{j} \underline{x}_{i j} \leq \bar{x}_{i o} i=1,2, \ldots, m \\
\sum_{j=1}^{n} \lambda_{j} \bar{y}_{r j} \geq \varphi \underline{y}_{r o}, r=1,2, \ldots, s \\
\sum_{j=1}^{n} \lambda_{j}=1 \\
\lambda_{j} \geq 0 \\
i=1,2, \ldots, m \quad, r=1,2, \ldots, s, j=1,2, \ldots, n
\end{gathered}
$$

In a completely similar way and regarding the lower bound of efficiency, pessimistic congestion is calculated by model (10):

$$
\begin{gathered}
\max \left(\sum_{\mathrm{r}=1}^{\mathrm{s}} \mathrm{s}_{\mathrm{r}}^{+}-\sum_{\mathrm{i}=1}^{\mathrm{m}} \mathrm{s}_{\mathrm{i}}^{-\mathrm{pc}}\right) \quad(10) \\
\mathrm{s.t}: \quad(60) \\
\sum_{\mathrm{j}=1}^{\mathrm{n}} \lambda_{\mathrm{j}} \underline{\mathrm{x}}_{\mathrm{ij}}+\mathrm{s}_{\mathrm{i}}^{-\mathrm{pc}}=\overline{\mathrm{x}}_{\mathrm{io}} \quad, \mathrm{i}=1,2, \ldots, \mathrm{m} \\
\sum_{\mathrm{j}=1}^{\mathrm{n}} \lambda_{\mathrm{j}} \overline{\mathrm{y}}_{\mathrm{rj}}-\mathrm{s}_{\mathrm{r}}^{+}=\underline{\mathrm{y}}_{\mathrm{ro}} \underline{\varphi}_{\mathrm{o}}^{*}, \mathrm{r}=1,2, \ldots, \mathrm{s} \\
\sum_{\mathrm{j}=1}^{\mathrm{n}} \lambda_{\mathrm{j}}=1 \\
\lambda_{\mathrm{j}} \geq 0, \mathrm{~s}_{\mathrm{r}}^{+} \geq 0, \mathrm{~s}_{\mathrm{i}}^{-\mathrm{pc}} \geq 0 \\
\mathrm{i}=1,2, \ldots, \mathrm{m}, \mathrm{r}=1,2, \ldots, \mathrm{s}, \mathrm{j}=1,2, \ldots, \mathrm{n}
\end{gathered}
$$


Therefore $s_{i}^{p c}$ is measured as pessimistic congestion. We know congestion is the result of inefficiency, but inefficiency does not necessarily lead to congestion. Inefficiency is a situation when to improve input and output becomes possible without even worsening some other input or output and this is different from the concept of congestion. Only based on the following conditions DMU is efficient:

1) $\max \varphi=\varphi^{*}=1$

2) $s_{r}^{*_{+}}=s_{i}^{*_{-}}=0 \quad \forall i, r$

\section{Case study}

At this case study 23 universities have been studied and the congestion value of each was employed both optimistically and pessimistically. The input and output data are as follows:

I1 = Faculty member

$\mathrm{I} 2=$ Students

$\mathrm{I} 3=$ Total current budget

I4 = Research budget

$\mathrm{O} 1=$ Books \& Articles

$\mathrm{O} 2=$ Master's degree admission

$\mathrm{O} 3=\mathrm{GPA}$ of students

Triangular inputs and outputs are shown in Table 1. The optimistic congestion value have been calculated based on formula (8), considering $\alpha=0.2, \alpha=0.5$ and $\alpha=0.7$ shown in Table 2, Table 3, Table 4 respectively. As seen, the pessimistic congestion value with $\alpha=0.2, \alpha=0.5, \alpha=0.7$ based on formula (10) are shown in Table 5, Table 6 and Table 7, in the order given.

Table 1.Fuzzy inputs \& outputs

\begin{tabular}{|c|c|c|c|c|c|c|c|}
\hline & $\mathbf{I}_{1}$ & $\mathbf{I}_{2}$ & $\mathbf{I}_{3}$ & $\mathbf{I}_{4}$ & $\mathbf{O}_{1}$ & $\mathbf{O}_{2}$ & $\mathbf{O}_{3}$ \\
\hline DMU1 & $\begin{array}{l}(0.10,0.16,0.18) \\
\end{array}$ & $(0.22,0.27,0.30)$ & $(0.47,0.62,1)$ & $\begin{array}{l}(2.57,3.47,5.28) \\
\end{array}$ & $(0.06,0.09,0.12)$ & $(0.01,0.01,0.04)$ & $\begin{array}{l}(0.15,0.16,0.26) \\
\end{array}$ \\
\hline DMU2 & $(0.26,0.28,0.32)$ & $(0.30,0.37,0.45)$ & $(0.43,0.46,0.56)$ & $(3.39,4.80,6.04)$ & $(0.08,0.16,0.42)$ & $(0.15,0.21,0.33)$ & $(0.35,0.38,0.44)$ \\
\hline DMU3 & $(0.12,0.17,0.20)$ & $(0.17,0.29,0.41)$ & $(0.16,0.23,0.26)$ & $(2.67,4.00,5.32)$ & $(0.06,0.08,0.13)$ & $(0.02,0.03,0.05)$ & $(0.10,0.14,0.17)$ \\
\hline DMU4 & $\begin{array}{l}(0.11,0.16,0.19) \\
\end{array}$ & $\begin{array}{l}(0.24,0.30,0.33) \\
\end{array}$ & $(0.57,0.65,0.75)$ & $(3,3.84,5.10)$ & $(0.06,0.08,0.11)$ & $(0.04,0.08,0.13)$ & $(0.14,0.20,0.26)$ \\
\hline DMU5 & $(0.16,0.28,0.32)$ & $(0.31,0.37,0.42)$ & $(0.57,0.60,0.74)$ & $(5.38,7.00,7.37)$ & $(0.12,0.14,0.17)$ & $(0.03,0.04,0.06)$ & $(0.43,0.46,0.52)$ \\
\hline DMU6 & $(1,1,1)$ & $(1,1,1)$ & $(0.81,0.90,1)$ & $(17.8,20.1,29.7)$ & $(1,1,1)$ & $(1,1,1)$ & $(1,1,1)$ \\
\hline DMU7 & $\begin{array}{l}(0.07,0.13,0.15) \\
\end{array}$ & $(0.24,0.26,0.29)$ & $\begin{array}{l}(0.51,0.57,0.59) \\
\end{array}$ & $(2.24,2.56,2.99)$ & $\begin{array}{l}(0.08,0.09,0.11) \\
\end{array}$ & $(0.03,0.05,0.14)$ & $\begin{array}{l}(0.14,0.19,0.25) \\
\end{array}$ \\
\hline DMU8 & $(0.07,0.12,0.14)$ & $(0.25,0.30,0.42)$ & $(0.22,0.46,0.49)$ & $(2.96,4.10,5.14)$ & $(0.02,0.04,0.08)$ & $(0.03,0.06,0.13)$ & $(0.13,0.17,0.21)$ \\
\hline DMU9 & $(0.16,0.22,0.24)$ & $(0.44,0.51,0.63)$ & $(0.34,0.58,0.71)$ & $(3.73,4.54,5.27)$ & $(0.07,0.13,0.17)$ & $(0.04,0.05,0.05)$ & $(0.21,0.28,0.36)$ \\
\hline DMU10 & $\begin{array}{l}(0.18,0.34,0.38) \\
\end{array}$ & $(0.32,0.33,0.37)$ & $(0.32,0.34,0.42)$ & $(5.12,7.99,12.9)$ & $(0.10,0.18,0.24)$ & $(0.03,0.32,0.48)$ & $(0.32,0.35,0.39)$ \\
\hline DMU11 & $(0.20,0.25,0.30)$ & $(0.28,0.35,0.56)$ & $(0.21,0.23,0.27)$ & $(4.64,5.72,6.32)$ & $(0.08,0.16,0.22)$ & $(0.08,0.13,0.22)$ & $(0.22,0.29,0.37)$ \\
\hline DMU12 & $(0.20,0.36,0.42)$ & $(0.35,0.39,0.50)$ & $(0.66,0.75,0.88)$ & $(3.91,6.10,10.9)$ & $(0.37,0.53,0.69)$ & $(0.04,0.09,0.23)$ & $(0.44,0.48,0.53)$ \\
\hline DMU13 & $(0.20,0.39,0.41)$ & $\begin{array}{l}(0.47,0.53,0.59) \\
\end{array}$ & $\begin{array}{l}(0.65,0.71,0.74) \\
\end{array}$ & $(5.38,6.72,10.6)$ & $\begin{array}{l}(0.14,0.20,0.26) \\
\end{array}$ & $(0.08,0.23,0.51)$ & $\begin{array}{l}(0.45,0.49,0.60) \\
\end{array}$ \\
\hline DMU14 & $(0.15,0.17,0.18)$ & $(0.19,0.23,0.28)$ & $(0.42,0.45,0.47)$ & $(3.41,4.16,5.10)$ & $(0.08,0.10,0.12)$ & $(0.04,0.06,0.09)$ & $(0.18,0.23,0.31)$ \\
\hline DMU15 & $(0.10,0.18,0.22)$ & $(0.31,0.35,0.38)$ & $(0.38,0.45,0.49)$ & $(3.94,5.05,5.41)$ & $(0.04,0.08,0.10)$ & $(0.01,0.05,0.08)$ & $(0.15,0.27,0.34)$ \\
\hline DMU16 & $(0.11,0.14,0.17)$ & $(0.19,0.22,0.27)$ & $(0.67,0.93,1)$ & $(2.98,3.69,4.89)$ & $(0.03,0.05,0.06)$ & $(0.02,0.05,0.10)$ & $(0.14,0.19,0.28)$ \\
\hline DMU17 & $(0.02,0.06,0.06)$ & $(0.12,0.14,0.17)$ & $(0.17,0.32,0.51)$ & $(1.80,2.46,4.68)$ & $(0.02,0.02,0.03)$ & $(0.00,0.02,0.03)$ & $(0.02,0.09,0.13)$ \\
\hline DMU18 & $(0.04,0.05,0.05)$ & $(0.07,0.10,0.14)$ & $(0.20,0.32,0.39)$ & $(1.10,1.38,1.70)$ & $(0.00,0.00,0.01)$ & $(0.00,0.01,0.03)$ & $(0.01,0.03,0.04)$ \\
\hline DMU19 & $(0.06,0.08,0.10)$ & $\begin{array}{l}(0.12,0.20,0.22) \\
\end{array}$ & $\begin{array}{l}(0.50,0.57,0.61) \\
\end{array}$ & $(2.06,2.30,2.66)$ & $\begin{array}{l}(0.01,0.02,0.03) \\
\end{array}$ & $(0.00,0.01,0.04)$ & $\begin{array}{l}(0.05,0.08,0.13) \\
\end{array}$ \\
\hline DMU20 & $(0.07,0.09,0.10)$ & $(0.07,0.18,0.20)$ & $(0.40,0.44,0.48)$ & $(2.44,3.26,4.34)$ & $(0.02,0.03,0.04)$ & $(0.01,0.08,0.13)$ & $(0.10,0.11,0.11)$ \\
\hline DMU21 & $(0.04,0.05,0.05)$ & $(0.07,0.08,0.11)$ & $(0.42,0.77,1)$ & $(1.55,1.69,1.97)$ & $(0.01,0.01,0.03)$ & $(0.00,0.00,0.00)$ & $(0.04,0.06,0.07)$ \\
\hline DMU22 & $(0.09,0.10,0.10)$ & $(0.25,0.33,0.41)$ & $(0.04,0.14,0.30)$ & $(2.44,3.02,3.63)$ & $(0.00,0.01,0.01)$ & $(0.01,0.01,0.02)$ & $(0.00,0.03,0.08)$ \\
\hline DMU23 & $(0.10,0.16,0.18)$ & $(0.13,0.20,0.24)$ & $(0.47,0.62,1)$ & $(2.00,2.84,3.09)$ & $(0.03,0.04,0.06)$ & $(0.00,0.01,0.02)$ & $(0.06,0.10,0.13)$ \\
\hline
\end{tabular}


Table 2.The result of optimistic congestion

\begin{tabular}{|c|c|c|c|c|c|c|c|c|}
\hline$\alpha=0.2$ & $\varphi^{*}$ & $s_{1}^{o c}$ & $s_{2}^{o c}$ & $s_{3}^{o c}$ & $s_{4}^{o c}$ & $s_{1}^{+*}$ & $S_{2}^{+*}$ & $s_{3}^{+*}$ \\
\hline DMU1 & - & - & - & - & - & - & - & - \\
\hline DMU2 & - & - & - & - & - & - & - & - \\
\hline DMU3 & - & - & - & - & - & - & - & - \\
\hline DMU4 & - & - & - & - & - & - & - & - \\
\hline DMU5 & 0.24 & 0 & 0 & 0 & 0.63 & 0.01 & 0.01 & 1 \\
\hline DMU6 & 0.44 & 0.45 & 0.35 & 0 & 3.1 & 0 & 0 & 0 \\
\hline DMU7 & 0.26 & - & - & - & - & - & - & - \\
\hline DMU8 & - & - & - & - & - & - & - & - \\
\hline DMU9 & - & - & - & - & - & - & - & - \\
\hline DMU10 & - & - & - & - & - & - & - & - \\
\hline DMU11 & - & - & - & - & - & - & - & - \\
\hline DMU12 & 0.2 & - & - & - & - & - & - & - \\
\hline DMU13 & 0.26 & 0.02 & 0.04 & 0 & 0 & 0.03 & 0 & 0.01 \\
\hline DMU14 & - & - & - & - & - & - & - & - \\
\hline DMU15 & - & - & - & - & - & - & - & - \\
\hline DMU16 & 0.37 & 0 & 0 & 0 & 0 & 0 & 0 & 0 \\
\hline DMU17 & - & - & - & - & - & - & - & - \\
\hline DMU18 & - & - & - & - & - & - & - & - \\
\hline DMU19 & - & - & - & - & - & - & - & - \\
\hline DMU20 & - & - & - & - & - & - & - & - \\
\hline DMU21 & - & - & - & - & - & - & - & - \\
\hline DMU22 & - & - & - & - & - & - & - & - \\
\hline DMU23 & - & - & - & - & - & - & - & - \\
\hline
\end{tabular}

Table 3. The result of optimistic congestion

\begin{tabular}{|c|c|c|c|c|c|c|c|c|}
\hline$\alpha=0.5$ & $\varphi^{*}$ & $S_{1}^{o c}$ & $s_{2}^{o c}$ & $S_{3}^{o c}$ & $S_{4}^{o c}$ & $S_{1}^{+*}$ & $S_{2}^{+*}$ & $S_{3}^{+*}$ \\
\hline DMU1 & 0.72 & 0 & 0 & 0 & 0 & 0 & 0.01 & 0 \\
\hline DMU2 & 0.39 & 0.09 & 0.09 & 0 & 0 & 0 & 0 & 0 \\
\hline DMU3 & - & - & - & - & - & - & - & - \\
\hline DMU4 & 0.66 & 0 & 0.04 & 0 & 0 & 0 & 0 & 0 \\
\hline DMU5 & 0 & 0.2 & 0 & 0.14 & 0 & 0 & 0 & 0 \\
\hline DMU6 & 0.74 & 0.21 & 0.17 & 0.02 & 0 & 0 & 0 & 0.06 \\
\hline DMU7 & 0.4 & 0 & 0.07 & 0.01 & 0 & 0 & 0 & 0 \\
\hline DMU8 & 0.33 & 0 & 0.06 & 0 & 0.88 & 0 & 0 & 0 \\
\hline DMU9 & 0.65 & 0 & 0.05 & 0 & 0 & 0.03 & 0.05 & 0.07 \\
\hline DMU10 & 0.27 & 0.01 & 0 & 0 & 0 & 0.04 & 0 & 0.12 \\
\hline DMU11 & - & - & - & - & - & - & - & - \\
\hline DMU12 & 0.37 & 0.04 & 0.02 & 0.03 & 0 & 0 & 0 & 0.09 \\
\hline DMU13 & 0.57 & 0 & 0 & 0.1 & 0.15 & 0.02 & 0 & 0.03 \\
\hline DMU14 & 0.57 & 0.01 & 0 & 0 & 0 & 0 & 0.01 & 0 \\
\hline DMU15 & 0.47 & 0 & 0.01 & 0 & 0 & 0 & 0.01 & 0 \\
\hline DMU16 & 0.65 & 0 & 0.01 & 0.03 & 0 & 0.02 & 0 & 0 \\
\hline DMU17 & - & - & - & - & - & - & - & - \\
\hline DMU18 & 0.55 & - & - & - & - & - & - & - \\
\hline DMU19 & 0.16 & 0 & 0 & 0 & 0 & 0.01 & 0 & 0.04 \\
\hline DMU20 & - & - & - & - & - & - & - & - \\
\hline DMU21 & - & - & - & - & - & - & - & - \\
\hline DMU22 & - & - & - & - & - & - & - & - \\
\hline DMU23 & 0.78 & - & - & - & - & - & - & - \\
\hline
\end{tabular}


Table 4.The result of optimistic congestion

\begin{tabular}{|c|c|c|c|c|c|c|c|c|}
\hline$\alpha=0.7$ & $\varphi^{*}$ & $S_{1}^{o c}$ & $S_{2}^{o c}$ & $S_{3}^{o c}$ & $S_{4}^{o c}$ & $S_{1}^{+*}$ & $S_{2}^{+^{*}}$ & $S_{3}^{+*}$ \\
\hline DMU1 & 0.61 & 0.01 & 0 & 0 & 0 & 0 & 0.01 & 0 \\
\hline DMU2 & 0.1 & 0.14 & 0 & 0 & 0.73 & 0 & 0 & 0.01 \\
\hline DMU3 & - & - & - & - & - & - & - & - \\
\hline DMU4 & 0.72 & 0 & 0 & 0 & 0 & 0 & 0 & 0 \\
\hline DMU5 & 0.51 & 0 & 0 & 0 & 0.09 & 0.02 & 0.06 & 0 \\
\hline DMU6 & 0.59 & - & - & - & - & - & - & - \\
\hline DMU7 & 0.30 & 0.02 & 0 & 0 & 0 & 0 & 0 & 0 \\
\hline DMU8 & - & - & - & - & - & - & - & - \\
\hline DMU9 & 0.45 & 0.03 & 0.14 & 0 & 0 & 0 & 0.01 & 0 \\
\hline DMU10 & - & - & - & - & - & - & - & - \\
\hline DMU11 & - & - & - & - & - & - & - & - \\
\hline DMU12 & 0.41 & 0.04 & 0 & 0 & 0 & 0 & 0 & 0.06 \\
\hline DMU13 & 0.65 & 0.03 & 0.03 & 0 & 0 & 0.04 & 0 & 0 \\
\hline DMU14 & - & - & - & - & - & - & - & - \\
\hline DMU15 & 0.44 & - & - & - & - & - & - & - \\
\hline DMU16 & 0.79 & 0 & 0 & 0 & 0 & 0.07 & 0 & 0.11 \\
\hline DMU17 & - & - & - & - & - & - & - & - \\
\hline DMU18 & - & - & - & - & - & - & - & - \\
\hline DMU19 & 0.30 & - & - & - & - & - & - & - \\
\hline DMU20 & - & - & - & - & - & - & - & - \\
\hline DMU21 & - & - & - & - & - & - & - & - \\
\hline DMU22 & - & - & - & - & - & - & - & - \\
\hline DMU23 & - & - & - & - & - & - & - & - \\
\hline
\end{tabular}

Table 5. The result of pessimistic congestion

\begin{tabular}{|c|c|c|c|c|c|c|c|c|}
\hline$\alpha=0.2$ & $\varphi^{*}$ & $s_{1}^{p c}$ & $s_{2}^{p c}$ & $S_{3}^{p c}$ & $s_{4}^{p c}$ & $s_{1}^{+^{*}}$ & $S_{2}^{+*}$ & $S_{3}^{+*}$ \\
\hline DMU1 & 1.9 & 0.12 & 0.05 & 0.46 & 0.3 & 0 & 0 & 0 \\
\hline DMU2 & 1.62 & - & - & - & - & - & - & - \\
\hline DMU3 & - & - & - & - & - & - & - & - \\
\hline DMU4 & 1.89 & 0.08 & 0 & 0.2 & 2.2 & 0.67 & 0.92 & 0 \\
\hline DMU5 & 1.17 & 0 & 0 & 0 & 0.29 & 0.17 & 0.32 & 0 \\
\hline DMU6 & 0.92 & 0.07 & 0.06 & 0 & 10.85 & 0 & 0 & 0 \\
\hline DMU7 & 2.2 & 0 & 0.04 & 0.02 & 0 & 0.06 & 0.08 & 0 \\
\hline DMU8 & 2.1 & 0 & 0.16 & 0 & 1.03 & 0.07 & 0.17 & 0 \\
\hline DMU9 & 2.02 & 0 & 0.25 & 0 & 0.05 & 0.09 & 0.2 & 0 \\
\hline DMU10 & 0.78 & - & - & - & - & - & - & - \\
\hline DMU11 & - & 0.17 & 0.08 & 0 & 5.2 & 0.02 & 0.2 & 0 \\
\hline DMU12 & 1.18 & 0 & 0 & 0 & 1.5 & 0.2 & 0.4 & 0 \\
\hline DMU13 & 1.25 & - & - & - & - & - & - & - \\
\hline DMU14 & 1.54 & 0.03 & 0.01 & 0 & 5.6 & 0.02 & 0.2 & 0 \\
\hline DMU15 & 1.88 & 0.01 & 0.07 & 0 & 0.33 & 0.92 & 0.34 & 0 \\
\hline DMU16 & 2.6 & 0.03 & 0 & 0.3 & 0.82 & 0.17 & 0 & 0 \\
\hline DMU17 & 6.1 & - & - & - & - & - & - & - \\
\hline DMU18 & - & - & - & - & - & - & - & - \\
\hline DMU19 & 4.5 & 0 & 0.02 & 0.09 & 0.02 & 0.2 & 0.27 & 0 \\
\hline DMU20 & 2.6 & - & - & - & - & - & - & - \\
\hline DMU21 & 3.25 & 0.01 & 0.03 & 0.51 & 0.3 & 0 & 0 & 0 \\
\hline DMU22 & 1 & 0.01 & 0.13 & 0 & 0.1 & 0 & 0 & 0.06 \\
\hline DMU23 & 4.5 & 0 & 0 & 0.11 & 0 & 0.04 & 0.04 & 0 \\
\hline
\end{tabular}


Table 6.The result of pessimistic congestion

\begin{tabular}{|c|c|c|c|c|c|c|c|c|}
\hline $\boldsymbol{\alpha}=\mathbf{0 . 5}$ & $\varphi^{*}$ & $s_{1}^{p c}$ & $s_{2}^{p c}$ & $s_{3}^{p c}$ & $s_{4}^{p c}$ & $s_{1}^{+^{*}}$ & $s_{2}^{+^{*}}$ & $\boldsymbol{S}_{3}^{+^{*}}$ \\
\hline DMU1 & 2.29 & 0 & 0 & 0 & 0.23 & 0.31 & 0.06 & 0 \\
\hline DMU2 & 1.28 & 0 & 0 & 0 & 0 & 0.12 & 0.07 & 0 \\
\hline DMU3 & 2.26 & 0 & 0.06 & 0 & 0.07 & 0 & 0.07 & 0 \\
\hline DMU4 & 2.22 & 0 & 0 & 0.11 & 0 & 0.31 & 0 & 0 \\
\hline DMU5 & 1 & 0 & 0 & 0.07 & 0 & 0.25 & 0.15 & 0.04 \\
\hline DMU6 & 0.09 & 0 & 0 & 0.09 & 5.93 & 0.91 & 0.91 & 0.91 \\
\hline DMU7 & 1.55 & 0 & 0.01 & 0.48 & 0 & 0.72 & 0.06 & 0 \\
\hline DMU8 & 1.88 & 0 & 0.05 & 0 & 0.91 & 0.4 & 0 & 0 \\
\hline DMU9 & 1.82 & 0 & 0.2 & 0.71 & 0 & 0.35 & 0.03 & 0 \\
\hline DMU10 & 1.24 & 0.09 & 0 & 0 & 3.9 & 0.03 & 0.03 & 0 \\
\hline DMU11 & 1.39 & 0.13 & 0.09 & 0 & 0.19 & 0.04 & 0.06 & 0 \\
\hline DMU12 & 1.22 & 0.02 & 0 & 0.11 & 1.30 & 0 & 0.17 & 0 \\
\hline DMU13 & 1.29 & 0 & 0.02 & 0.04 & 0 & 0.15 & 0.17 & 0 \\
\hline DMU14 & 1.62 & 0.01 & 0 & 0 & 0 & 0.04 & 0 & 0 \\
\hline DMU15 & 1.95 & 0 & 0.06 & 0 & 0 & 0.01 & 0.02 & 0 \\
\hline DMU16 & 1.98 & 0 & 0 & 0.4 & 0 & 0.4 & 0.04 & 0.14 \\
\hline DMU17 & 1.12 & 0 & 0.02 & 1.11 & 0.2 & 0.13 & 0.04 & 0.05 \\
\hline DMU18 & 2.71 & 0 & 0 & 0.02 & 0 & 0.2 & 0.01 & 0 \\
\hline DMU19 & 2.91 & 0 & 0 & 0.03 & 0 & 0.59 & 0.07 & 0 \\
\hline DMU20 & 1.84 & 0 & 0 & 0 & 0.88 & 0.09 & 0 & 0 \\
\hline DMU21 & 1.50 & 0 & 0 & 0.04 & 0.03 & 0 & 0.06 & 0.01 \\
\hline DMU22 & 8.27 & 0 & 0.15 & 0 & 0.19 & 0 & 0 & 0 \\
\hline DMU23 & 2.52 & 0 & 0 & 0.21 & 0.02 & 0.35 & 0.03 & 0 \\
\hline
\end{tabular}

Table 7. The result of pessimistic congestion

\begin{tabular}{|c|c|c|c|c|c|c|c|c|}
\hline$\alpha=0.7$ & $\varphi^{*}$ & $s_{1}^{p c}$ & $S_{2}^{p c}$ & $S_{3}^{p c}$ & $S_{4}^{p c}$ & $S_{1}^{+^{*}}$ & $S_{2}^{+*}$ & $s_{3}^{+^{*}}$ \\
\hline DMU1 & 1.91 & 0 & 0 & 0 & 0 & 0.02 & 0.03 & 0 \\
\hline DMU2 & 0.67 & 0.06 & 0.06 & 0 & 0 & 0.04 & 0.2 & 0 \\
\hline DMU3 & - & - & - & - & - & - & - & - \\
\hline DMU4 & 1.64 & 0 & 0.05 & 0 & 0.12 & 0 & 0 & 0 \\
\hline DMU5 & 0.89 & 0 & 0.04 & 0 & 0 & 0.09 & 0.26 & 0 \\
\hline DMU6 & 0.76 & 0.21 & 0.16 & 0 & 7.4 & 0 & 0.01 & 0.01 \\
\hline DMU7 & 1.08 & 0 & 0.05 & 0 & 0 & 0 & 0.05 & 0 \\
\hline DMU8 & 0.97 & 0 & 0.08 & 0 & 0.7 & 0.02 & 0.06 & 0 \\
\hline DMU9 & 1.31 & 0 & 0.24 & 0 & 0 & 0.02 & 0.15 & 0 \\
\hline DMU10 & 0.67 & - & - & - & - & - & - & - \\
\hline DMU11 & - & - & - & - & - & - & - & - \\
\hline DMU12 & 0.96 & 0.01 & 0 & 0 & 0.39 & 0 & 0.18 & 0 \\
\hline DMU13 & 1.01 & 0 & 0.1 & 0 & 0 & 0 & 0.21 & 0.18 \\
\hline DMU14 & 0.74 & 0.02 & 0 & 0 & 0.72 & 0.02 & 0.02 & 0 \\
\hline DMU15 & 0.87 & 0 & 0.06 & 0 & 0.31 & 0.04 & 0.15 & 0 \\
\hline DMU16 & 1.56 & 0 & 0 & 0.22 & 0 & 0.03 & 0 & 0 \\
\hline DMU17 & - & - & - & - & - & - & - & - \\
\hline DMU18 & - & - & - & - & - & - & - & - \\
\hline DMU19 & 2.21 & 0 & 0.02 & 0 & 0 & 0.02 & 0.04 & 0 \\
\hline DMU20 & 0.66 & 0.01 & 0 & 0 & 1.2 & 0 & 0 & 0 \\
\hline DMU21 & 1.66 & - & - & - & - & - & - & - \\
\hline DMU22 & - & - & - & - & - & - & - & - \\
\hline DMU23 & 2.9 & 0 & 0.01 & 0 & 0 & 0.05 & 0.01 & 0 \\
\hline
\end{tabular}

In some universities, as observed in the given tables, there is congestion in some inputs; for example, in DMU6 in the optimistic case and in the first input there is congestion measuring up to 0.45 and in the pessimistic case 0.07 and these figures for the second input are consecutively 0.35 and 0.06 . Also for the fourth input the figures are consecutively 3.1 and 10.85 which reveals that if the value of congestion is decreased from the inputs the result will still be the same output. 


\section{Conclusion}

This article dealt with the calculation of congestion in data envelopment analysis with fuzzy inputs and outputs based on the Cooper's single model method. We have developed this method with inputs and outputs as fuzzy numbers. DMUs inputs and outputs can be regarded as triangular or trapezoidal fuzzy numbers where the triangular fuzzy numbers are taken into account. After applying $\alpha$ - cut approach, the lower bound and upper bound for input, output and efficiency is calculated and finally congestion is evaluated in optimistic and pessimistic viewpoint. As seen in concepts, congestion in optimistic view point takes in the minimum input and produces the optimum output. On the other hand, congestion in pessimistic viewpoint takes in the maximum input and gives out worst output. In these two models, the under-investigation DMU is in binary opposition with other DMUs. Finally, by studying an example, it is seen that the presence and absence of congestion in pessimistic and optimistic view point are completely separate. For further research suggestion, this proposed method can be applied with stochastic inputs and outputs or grey numbers to calculate congestion in optimistic and pessimistic viewpoints. Also, the above model can be changed into fuzzy model with the methods which were suggested earlier in FDEA. Another suggestion is to combine the method proposed earlier for the evaluation of congestion in the classical DEA with our method for the evaluation of congestion in optimistic and pessimistic viewpoint. Finally, the presence and the absence of congestion in optimistic and pessimistic viewpoint can be tested by statistical analyses, and the results can then be applied. The congestion model proposed in this article can also be employed to improve management in other institutes and organizations.

\section{References}

[1] A. Charnes, W.W. Cooper, E. Rhodes, Measuring the efficiency of decision making units, European Journal of the Operational research, (1978) 2.429-444.

[2] R.D. Banker, A. Charnes, W.W. Cooper, Some model for estimating technical and scale inefficiencies in data envelopment analysis, Management science, (1984) 30 1078-1092.

[3] A. Alinezhad, M. Zohrebandian N. Esfandiary, Introduction on performance measurement systems, Azad university of Qazvin, Publication of Azad University, (2011).

[4] W.W. Cooper, K.S. Park, G. Yu, IDEA and AR-IDEA, Models for dealing with imprecise data in DEA, Management Science, (1999) 45, 597-607.

[5] W.W. Cooper, H. Deng, B. Gu, S.X. Li, R.M. Thrall, Using DEA to improve the management of congestion in Chinese industries, Socio- Economic Planning Science, (2001) 35, 1981-1997.

[6] J. Zhu, Imprecise data envelopment analysis (IDEA) A review and improvement with an application, European Journal of Operational Research, (2003) 144, 513-529.

[7] L. Pour Karimi, Introduction of congestion concept based on production function in data envelopment analysis, MSc thesis, applied mathematics (Operation research), Tarbiat Moalem University (2005).

[8] R. Fare, S. Grosskopf, CAK. Lovell, The measurement of efficiency of production Boston, MN:Klewer-Nijhoff publishing, (1985).

[9] W.W. Cooper, R.G. Thompson, R.M. Thrall, Introductions and new developments in DEA, Annals of Operations Research, (1996) 66 3-45.

[10] H. Zarehaghighi, Study on congestion evaluation methods in data envelopment analysis, Applied mathematics (operation research), MSc thesis, Tarbiat MoalemUniversity, (2010).

[11] P. L. Brockett, W.W. Cooper, H.C, Shin, Y. Wang, Inefficiency and congestion in Chinese production before and after the 1978 economic reforms, Socio-Economic Planning Sciences, (1988) 3, 1-20.

[12] W.W. Cooper, L.M. Seiford, J. Zhu, A unified additive model approach for evaluating efficiency and congestion with associated measures in DEA, Socio-Economic Planning Sciences, (2000) 34, 1-25.

[13] Tone, K., Sahoo, B. K. (2004). Degree of scale economics and Congestion: A unified DEA approach. European Journal of Operational Research, 158, 755-772.

[14] G. R. Jahanshahloo, M. Khodabakhshi, Suitable combination of input for improving output in DEA with determining input congestion considering textile industry of china, Applied Mathematics and Computation, (2004) 151, 263-273.

[15] M. Khodabakhshi,A one model approach based on relaxed combination of inputsforevaluatinginputcongestioninDEA, JournalofcomputationalApplied Mathematics, (2009) 230, 443-450. 
[16] S. Fanati Rashidi, A new method for measuring congestion in data envelopment analysis, PHD thesis, Azad university of OloomTahghighat(Tehran), (2010).

[17] L.A. Zadeh, Fuzzy sets, Inform and control, (1965) 338-353.

[18] M. YazdanPanahMariki, Congestion calculating in Fuzzy data envelopment analysis, calculating in Fuzzy data envelopment analysis, MSc thesis, Applied Mathematics, Azad university of Broujerd (2012).

[19] M.S. Saati, A. Memariani, G.R. Jahanshahloo, Efficiency analysis and Ranking of DMUs with Fuzzy Data. Fuzzy Optimization and Decision Making, (2002) 255-267.

[20] W.W. Cooper, B. Gu, S. Li, Note:Alternative treatments of congestion in DEA a response to the Cherchye, Kuosmonen and post critique, European journal of operational research, (2001a) 132, 81-87. 